\title{
Pharmacogenomic (PGx) Testing as a Standard of Clinical Care
}

\author{
Michael J Schuh* and Judith S Kaur \\ Mayo Clinic School of Health Sciences, College of Medicine, USA
}

*Corresponding author: Michael J Schuh, Mayo Clinic School of Health Sciences, College of Medicine, Jacksonville, Florida, USA

\begin{tabular}{|c|c|}
\hline ARTICLE INFO & ABSTRACT \\
\hline 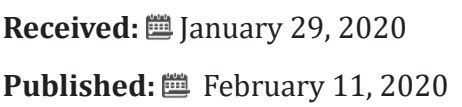 & $\begin{array}{l}\text { Citation: Michael J Schuh, Judith S Kaur. Pharmacogenomic (PGx) Testing as a Standard } \\
\text { of Clinical Care. Biomed J Sci \& Tech Res 25(3)-2020. BISTR. MS.ID.004215. }\end{array}$ \\
\hline
\end{tabular}

\section{Opinion}

\section{Should Pharmacogenomics (PGx) Testing be a New Standard of Clinical Care in 2020 ?}

The human genome sequencing began in 1999 and was completed in 2000. That scientific accomplishment paved the way for understanding gene functions as they relate to clinical medicine. So, what is PGx testing and how can it help patients? PGx testing is a method of genetically determining how humans metabolize drugs. It is now possible to identify how well genes function that code for the pharmacokinetic cytochrome P-450 enzymes (also named CYP enzymes). PGx testing costs have been reduced from thousands of dollars for testing one or a few genes, to a few hundred dollars for an entire panel of genes. In addition, publication of evidencedbased literature validating the science is occurring at a very rapid rate $[1,2]$. We now understand how pharmacodynamic proteins work at medication target sites [3]. By using PGx testing we can start to eliminate the traditional trial and error method of drug selection to treat many diseases such as cancer. How is PGx testing performed? DNA samples are obtained using a buccal swab and the sample is sent to a certified clinical laboratory. Performing sample collection in a clinical setting utilizes established methods and procedures [4].

Although obtaining the sample is easy and the gene panel testing and reporting are relatively inexpensive, usually costing just a few hundred dollars, it takes some training to interpret and apply the testing results. A basic understanding of pharmacology and pharmacokinetics is required along with a very basic understanding of genetics [5]. The known literature reports varying levels of evidence for studied genes, therefore reporting of lab results to patients and determination of actionable results requires clinical judgment for interpreting and applying results. This can be an obstacle to obtaining third party payment for PGx panels as the panels include genes with strong, actionable evidence and genes with less than actionable evidence based on the strength of the available literature [3]. Good sources of the literature for test interpretation are the Clinical Pharmacogenetics Implementation Consortium (CPIC), Dutch Pharmacogenetics Working Group (DPWG) or a compendium of these and other sources such as the Pharmacogenomics Knowledgebase, at Stanford University [3].

What are the challenges regarding incorporation of PGx panel testing as a standard of care?

Awareness that this science may be quite valuable in many specialties is an important start. An important example is the integration of palliative care into standard oncology care. The American Society of Clinical Oncology Guidelines were developed from an Expert Panel of members in 2016 (https://www.asco. org/research-guidelines/quality-guidelines/guidelines/supportive-care-and-treatment-related-issues\#/9671). Pharmacogenomics can significantly aid in selection of pain management, nausea control, depression and even prevention of toxicity from certain chemotherapeutic agents. This can significantly improve patients' quality of life. Even in early stages PGx' $\mathrm{s}$ is a viable guide for medication therapy and clinical decision making. By helping to eliminate trial and error of drug selection by clinicians this data can decrease use of medications that may be ineffective for treatment and medications or even those that can cause serious drug interactions or adverse drug reactions (ADRs) that may be fatal, such as Stevens-Johnson syndrome [6]. 
The result can lead to lower health care costs with reduction of emergency room visits and hospital admissions. There may also be fewer episodes of failed therapy especially with some of the new very expensive medications $[7,8]$. Identifying patients who have the most benefit and least toxicity will improve patient care and make these powerful therapies more cost effective. Payers may still argue that there are varied levels of evidence available for different genes to make clinical decisions [3]. However, other more established tests also have varying levels of evidence, also requiring clinical interpretation as well that may not be definitive $[9,10]$.One suspects that as costs of these pharmacogenomic tests are reduced, they will become more widespread for clinical use. As clinicians and their supportive pharmacists gain comfort level in interpretation, they will value their effectiveness in improving patient care. Increased PGx formal education and payment for testing will move the needle to acceptance of this new standard of care [11,12]. Due to the prospect of better clinical outcomes and lower health care costs, PGx testing will become a standard of quality care in most medical settings in the very near future.

\section{References}

1. Moyer AM, Caraballo PJ (2017) The challenges of implementing pharmacogenomic testing in the clinic. Expert Review of Pharmacoeconomics \& Outcomes Research 17(6): 567-577.

2. PharmGKB (2020) Available at: https://www.pharmgkb.org/page/ history-PharmGKB History. Accessed Jan 13, 2020

3. Whirl Carrillo M, McDonagh EM, Hebert JM, Gong L, Sangkuhl K, et al. (2012) Pharmacogenomics Knowledge for Personalized Medicine. Clinical Pharmacology \& Therapeutics 92(4): 414-417.

\section{ISSN: 2574-1241}

DOI: $10.26717 /$ BJSTR.2020.25.004215

Michael J Schuh. Biomed J Sci \& Tech Res

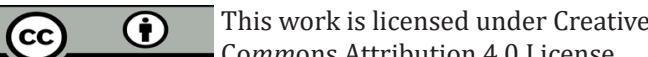

Submission Link: https://biomedres.us/submit-manuscript.php
4. Arwood MJ, Chumnumwat S, Cavallari LH, EA Nutescu, JD Duarte et al. (2016) Implementing Pharmacogenomics at Your Institution: Establishment and Overcoming Implementation Challenges. Clin Transl Sci 9(5): 233-245.

5. Just KS, Steffens M, Swen JJ, Jesse Joachim SwenGeorge P. Patrinos, et al. (2017) Medical education in pharmacogenomics-results from a survey on pharmacogenetic knowledge in healthcare professionals within the European pharmacogenomics clinical implementation project Ubiquitous Pharmacogenomics (U-PGx). Eur J Clin Pharmacol 73(10): 1247-1252.

6. Khor AH, Lim KS, Tan CT, Kwan Z, Tan WC, et al. (2017) HLA-A*31: 01 and HLA-B*15:02 association with Stevens-Johnson syndrome and toxic epidermal necrolysis to carbamazepine in a multiethnic Malaysian population. Pharmacogenet Genomics 27(7): 275-278.

7. Maciel A, Cullors A, Lukowiak AA, Garces J (2018) Estimating cost savings of pharmacogenetic testing for depression in real-world clinical settings. Neuropsychiatr Dis Treat 14: 225-230.

8. Krebs K, Milani L (2019) Translating pharmacogenomics into clinical decisions: do not let the perfect be the enemy of the good. Hum Genomics 13: 39.

9. Conrad K, Röber N, Andrade LE, Mahler M (2017) The Clinical Relevance of Anti-DFS70 Autoantibodies. Clin Rev Allergy Immunol 52(2): 202216 .

10. Bray C, Bell LN, Liang H, Haykal R, Kaiksow F,et al. (2016) Erythrocyte Sedimentation Rate and C-reactive Protein Measurements and Their Relevance in Clinical Medicine. WMJ 115(6): 317-321.

11. Kuželički NK, Žitnik IP, Gurwitz D, Adrian Llerena, Ingolf Cascorbi, et al. (2019) Pharmacogenomics education in medical and pharmacy schools: conclusions of a global survey. Pharmacogenomics 20(9).

12. Clinicalomics. Available at: https://www.clinicalomics.com/topics/ molecular-dx-topic/unitedhealthcare-to-cover-genetic-testing-forprecision-medicine-in-depression-anxiety. Accessed Jan 20, 2020.

$\begin{array}{ll}\text { BIOMEDICAL } & \text { Assets of Publishing with us } \\ \text { RESEARCHES } & \text { - Global archiving of articles } \\ & \text { - Immediate, unrestricted online access } \\ & \text { - Rigorous Peer Review Process } \\ \end{array}$

